\title{
Food Waste and Byproduct Valorization through Bio-processing: Opportunities and Challenges
}

\author{
Xavier Turon, ${ }^{\mathrm{a}, *}$ Joachim Venus, ${ }^{\mathrm{b}}$ Mehrdad Arshadi, ${ }^{\mathrm{c}}$ Michalis Koutinas, ${ }^{\mathrm{d}}$ \\ Carol S.K. Lin, ${ }^{e}$ and Apostolis Koutinas ${ }^{\mathrm{f}, *}$ \\ The bioeconomy era will rely on efficient fractionation of renewable \\ resources via integrated biorefineries. The food supply chain waste, \\ despite its inherent variability, could evolve into an important industrial \\ feedstock on account of its availability, versatility, and sustainability, for \\ the production of bio-based products. Waste streams generated from all \\ stages of the life cycle of food products could be refined into different \\ fractions, which will be either purified to high-value molecules or converted \\ via green chemical and/or biotechnological routes for the production of bio- \\ based products. A working group of the EUBis COST Action TD1203 is \\ taking steps to gather a critical mass of knowledge and expertise to create \\ innovation and technological breakthroughs.
}

Keywords: Food supply chain waste; Biorefinery; Bioprocessing; Chemicals; Bio-based products

Contact information: a: Department of Bioengineering, IQS-Ramon Llull Univ. Via Augusta 390 Barcelona, Spain; $b$ : Dept. Bioengineering, Leibniz-Institute for Agricultural Engineering Potsdam-Bornim e.V., Potsdam, Germany; c: Dept. Forest Biomaterials and Technology, Swedish University of Agricultural Sciences. Umeå, Sweden; d: Dept. Environmental Science and Technology, Cyprus University of Technology. Limassol, Cyprus; e: School of Energy \& Environment, City University of Hong Kong, Hong Kong, China; f: Department of Food Science and Human Nutrition, Agricultural University of Athens, Athens, Greece; *Corresponding authors: xavier.turon@iqs.edu; akoutinas@aua.gr

\section{Food Supply Chain Waste}

Food supply chain waste (FSCW) is nowadays widely recognized as an immense environmental, economic, and societal burden that should be addressed through both prevention and valorization policies. The huge quantities of FSCW generated annually (1.3 billion tons of edible food or 1.6 billion tons of "primary product equivalents") is more than $20 \%$ of the total agricultural production for food and non-food uses that requires around $30 \%$ of the total agricultural land area (FAO 2013). Waste generated from noneaten food won the bronze medal in annual $\mathrm{CO}_{2}$ emissions ( 3.3 billion $\mathrm{t}$ ) after the nations USA and China (FAO 2013). The economic burden caused by the wastage of agricultural products meant to be used as food is close to the GDP of Switzerland (USD 750 billion) (FAO 2013). In Hong Kong, about 3,600 t of food waste is generated every day, accounting for almost $40 \%$ of municipal solid waste. With the waste-to-landfill approach, most of the food waste is disposed to landfills, losing a valuable resource and the opportunity of regeneration of carbon-rich feedstocks. In view of the closure of all landfills in Hong Kong by 2018 , there is an urgent need to divert food waste from landfills.

Food waste is generated in every stage along the life cycle of different food supply chains, starting from agricultural production and postharvest practices to industrial processing, wholesale/retail sector, food service sector, and household consumption. More than $60 \%$ of food waste mainly occurs at the retail, distribution, food service, and household sectors in developed countries. The less developed a country is, the greater the quantities of food lost due to poor harvest and processing practices. This also illustrates the 
societal dimension of food waste generation, where especially in developed countries, policies should target the improvement of consumer's attitude and awareness toward food consumption and waste generation. The impact of global climate change, the rapid economic development of emerging markets, and the gradual but imminent depletion of fossil fuel resources creates an opportunity for FSCW to emerge as a sustainable resource, providing renewable carbon for industrial production of chemicals, polymers, materials, energy, and fuels. FSCW was recently ranked third among 15 opportunities that could be used to address the resource challenge through expansion of supply and improvement of productivity (McKinsey GI Report 2011). Converting FSCW into an industrial feedstock cannot be achieved by conventional food waste management based mainly on landfilling or conventional recycling practices (e.g. composting, animal feed). FSCW should be initially divided into avoidable and unavoidable fractions. New policies and innovative technologies should be developed to reduce the former fraction and to exploit the latter fraction through sustainable biorefineries (Lin et al. 2013; Koutinas et al. 2014). In Europe, the EUBis COST Action TD 1203 works to develop novel strategies for the valorization of FSCW into new, sustainable, and functional feedstocks. The Action has working groups based on pre-treatment and extraction of value-added compounds, bio-processing, green chemical processing, and sustainability assessment/policy analysis. This strategy could be applied in a similar manner in other parts of the world.

This Editorial article focuses mainly on bioprocessing of FSCW for the production of platform chemicals, bio-based polymers, and fuels. Although bioprocessing was explored for chemical production (i.e., evaluation of 2,3-butanediol production in pilot scale fermentations during World War II as a precursor for synthesis of 1,3-butadiene, a monomer for synthetic rubber production) before the 1950s, the immense advantages of petroleum as a feedstock and the favorable economies of scale achieved by petroleum refineries outcompeted biomass and bioprocessing for chemical and polymer production. The enormous quantities of FSCW could provide an alternative industrial feedstock, and biorefining could provide the profitability margin required via initial fractionation of valueadded products, such as bioactive compounds for high-value applications. FSCW contain various polysaccharides (e.g. starch, lignocellulosics) that could be used as carbon sources in microbial bioconversions. The aim is either to develop direct microbial bioconversion such that FSCW can be assimilated without pre-treatment or the bioconversion of FSCW after pre-treatment involving enzymatic or chemical processes. A Working Group of the EUBis COST Action has been focusing on utilisation of existing and new methodologies for pre-processing and selective extraction of chemicals from food waste using green/no solvent methods and eco-friendly low-cost processes with minimal consumption of reagents, as well as chemical and physical characterisation of the raw and pre-treated materials with special emphasis on extraction technologies. The main objective is to identify cost-effective processes for combined state of the art characterization and extraction of high-value chemicals from selected FSCW. Various methods for isolation/separation of high value chemicals from food waste are used (e.g. supercritical fluids or microwave assisted extraction).

The development of sustainable fermentation processes can be achieved through maximum product concentration ( $\mathrm{kg}$ product per $\mathrm{m}^{3}$ fermentation broth), carbon source to product conversion yield ( $\mathrm{kg}$ product per $\mathrm{kg}$ carbon source), and productivity (kg product per $\mathrm{m}^{3}$ per $\mathrm{h}$ of fermentation). In recent years, there has been significant interest in the development of industrial fermentations including succinic acid production by BioAmber, 
CSF/BASF, Roquette/DSM, Myriant, and Lanxess; lactic acid by NatureWorks and Purac; polyhydroxybutyrate (PHB) by Telles and Tianjin GreenBio Material; microbial oil by Neste Oil, and 1,3-propanediol by Metabolic Explorer and Tate \& Lyle/DuPont, among others (Carus 2012). A Working Group of the EUBis consortium has been focusing on the evaluation of FSCW for the production of various fermentation products including platform chemicals, bio-based polymers, microbial oil, enzymes, biosurfactants, and biocolorants, among others. The main challenge will be to achieve high production yields, low processing costs, and feasible industrial scale-up. Some examples of fermentation processes are presented below.

Fermentation media derived from FSCW could be employed for production of triglycerides (TAGs) via cultivation of heterotrophic oleaginous microorganisms (Leiva et al. 2014). Microbial oil is accumulated intracellularly under nutrient-limiting conditions. Oleaginous yeasts consume various carbon sources, including C5 and C6 sugars, sucrose, lactose, and glycerol, whereas they can accumulate microbial lipids up to $70 \%$ of their dry cell weight. Specific fermentation media and conditions as well as microbial strain selection enable the production of microbial oils with different fatty acid composition, providing the opportunity for using such oils in diversified market outlets including biodiesel, oleochemical, medical, dietetical, and food related applications. Microbial oils could replace vegetable oils and fossil feedstock for the production of various surfactants, waxes, lubricants, cosmetics, and paints among others. Eukaryotic microorganisms are prolific producers of high value-added metabolites, such as omega-3 long chain polyunsaturated fatty acids, pigments, sterols, and terpenoids (Abad and Turon 2012). The production of such nutraceuticals, with relevant importance for adult, and baby health, will facilitate the economic viability of a biorefinery scenario (Turon 2013).

Platform chemical production via fermentation of FSCW could provide renewable feedstocks for development of a sustainable chemical industry. Various C2-C6 chemical intermediates could be produced via fermentation (Koutinas et al. 2014). Besides biofuel application, ethanol from FSCW could be used as a platform intermediate. Braskem (Brazil) is currently producing ethylene and propylene from bioethanol. Succinic acid is considered as one of the most promising platform chemicals that can be produced from FSCW through microbial fermentation. It can be either used directly or as a chemical intermediate to produce a wide variety of other value-added chemicals with numerous applications in food, pharmaceuticals, and chemical industries. A wide range of FSCW (e.g. fruit/vegetable or bakery waste streams, whey, wine/sake lees) has been previously employed for microbial production of succinic acid. Wild type and recombinant strains used have the capacity to fix carbon dioxide into succinic acid during fermentation, contributing to the reduction of greenhouse gas emissions. Succinic acid production could be achieved by both $\mathrm{C} 5$ and $\mathrm{C} 6$ sugars, sucrose, lactose, maltose, and glycerol. The theoretical yield could be up to $1.3 \mathrm{~g}$ succinic acid per g glucose due to $\mathrm{CO}_{2}$ fixation.

Bio-based polymer production from FSCW could lead to simultaneous waste management and production of packaging articles for food products. Some representative bio-based plastics are polyhydroxyalkanoates (PHA) accumulated intracellularly by various bacterial strains under nutrient limiting conditions and polylactic acid (PLA) derived from polymerization of lactic acid produced by fermentation. Lactic acid and PHA production from FSCW could be a suitable way for sustainable recycling of food waste. In the sense of closed loop process development, fully biodegradable PLA films could be produced from bakery waste. This novel approach will include conversion of organic 
bakery waste into fermentation feedstock for lactic acid production followed by polymerization of lactic acid to PLA, modification of PLA by compounding, film production and final testing of these films for packaging applications (Gonzalez and Siliba 2012). Similar approaches could be followed in the case of PHA-based packaging articles derived via fermentative recycling of FSCW.

FSCW could be also used for the production of industrial enzymes, biocolorants, biosurfactants, and bacterial cellulose. Natural pigments could be produced via fermentation of bakery waste using the fungal strain Monascus purpureus with applications in the textile and food industries. Sphorolipids are glycolipids composed of a sophorose disaccharide covalently attached to both ends of a medium-chain fatty acid (usually C16 or C18). Bacterial cellulose is a versatile and biocompatible polymer with numerous applications in food, medical, cosmetic, and biomaterial industries that could be produced by various carbon sources contained in FSCW using static or agitated cultures with acetobacteria such as Gluconacetobacter xylinus.

\section{Conclusion and Perspectives}

FSCW could supply a low-cost and readily available feedstock for the chemical industry, creating a smoother transition to the bio-economy era. The inherent variability of food waste remains a principle challenge. To address such challenges, integration of biorefinery concepts in traditional food industry plants for the valorization of food waste will create added-value and innovation for various industrial sectors. The food industry sector is dominated by SMEs and collaboration with the chemical industry sector is essential in order to implement the necessary research and development initiatives.

\section{ACKNOWLEDGEMENTS}

The authors acknowledge the contribution of the COST Action TD1203 - EUBis

\section{REFERENCES CITED}

Abad, S., and Turon, X. (2012). "Valorization of biodiesel derived glycerol as a carbon source to obtain added-value metabolites: Focus on polyunsaturated fatty acids," Biotech. Adv. 30(3), 733-741.

Carus, M. (2012). "World-wide investments in bio-based chemicals," www.biobased.eu/policy/

FAO. (2013). "Food wastage foodprint: Impact on natural resources. Summary report."

Gonzalez, R., and Siliba, M. A. (2012). "Bread4PLA," Bioplastics Magazine 7(4), 18-19.

Koutinas, A. A., Vlysidis, A., Pleissner D., Kopsahelis, N., et al. (2014). "Valorization of industrial waste and by-product streams via fermentation for the production of chemicals and biopolymers," Chem. Soc. Rev. 43(8), 2587-2627.

Leiva-Candia, D. E., Pinzi, S., Redel-Macías, M. D., Koutinas, A., et al. (2014). “The potential for agro-industrial waste utilization using oleaginous yeast for the production of biodiesel," Fuel 123, 33-42.

Lin, C. S. K., Pfaltzgraff, L.A., et al. (2013). "Food waste as a valuable resource for the production of chemicals, materials and fuels. Current situation and global perspective," Energy Environ. Sci. 6(2), 426-464.

McKinsey Global Inst. report. (2011). "Resource revolution: Meeting the world's needs."

Turon, X. (2013). "Algae oil, a valuable source of long chain polyunsaturated fatty acids," J. Life Med. 1(1), 11-14. 